Revista Colombiana de Obstetricia y Ginecología Vol. 56 No.2 • 2005 • (166-175)

ARTículo De Revisión

\title{
PACIENTE OBSTÉTRICA
}

\section{DE ALTO RIESGO: ¿DÓNDE DEBE REALIZARSE SU CUIDADO PERIPARTO?}

\author{
Where should peripartum care of high-risk obstetric \\ patients take place?
}

Juan Felipe Aristizábal, M.D.*, Germán Alberto Gómez, M.D.*, Juan Francisco Lopera, M.D.*, Laura Victoria Orrego M.D. *, Carlos Restrepo, M.D.**, Germán Monsalve, M.D.**, Martín Gómez M.D.**, Nury Socha, M.D.**, Mauricio Vasco, M.D.**

Recibido: febrero 21/05 - Revisado: mayo 10/05. - Aceptado: mayo 31/05

\section{RESUIMEN}

Este artículo presenta los patrones que determinaron el ingreso de las gestantes críticamente enfermas a la Unidad de Cuidados Intensivos (UCI) o de Alta Dependencia Obstétrica (UAD), y las causas de complicaciones y muerte más frecuentes, al igual que los factores que afectan el pronóstico perinatal.

Se plantea la implementación de las Unidades de Alta Dependencia Obstétrica para el manejo de las gestantes críticamente enfermas, y el entrenamiento del personal involucrado en el manejo obstétrico de estas pacientes.

Palabras clave: unidad de cuidados intensivos, unidad de alta dependencia obstétrica, embarazo.

\section{SUMMARY}

This article outlines the patterns determining critically ill pregnant women's admittance to an intensive care or high-dependency unit, the most frequently occurring causes of complications and death and the factors affecting perinatal prognosis.

* Residentes de Anestesia CES

** Anestesiólogos. Grupo de interés en anestesia obstétrica. GIAO, Comité Nacional de Anestesia Obstétrica. SCARE, Grupo de Investigación en Anestesiología y Reanimación. GUIAR. Universidad Pontificia Bolivariana, Medellín. Correo electrónico: machuchovasco@yahoo.com
High-dependency units would then seem to have become necessary for treating critically ill pregnant women. Suitable training of the personnel involved in these patients' obstetric care has also become a priority.

Key words: intensive care unit, obstetric highdependency unit, pregnancy.

\section{INTRODUCCIÓN}

La mortalidad materna es una preocupación a nivel mundial. Diariamente mueren unas 1.600 mujeres en el mundo por complicaciones relacionadas con el embarazo. ${ }^{1}$

Se ha definido una muerte materna como aquélla que se produce durante el embarazo y hasta un año después del parto, y que es consecuencia de éste, evidenciándose en los últimos tiempos un incremento en algunas de sus causas, principalmente por enfermedades crónicas como las cardiomiopatías ${ }^{2}$ (tabla 1).

Existe una gran discrepancia en las estadísticas de mortalidad entre los países desarrollados y subdesarrollados, las cuales están muy relacionadas con situaciones socioeconómicas claramente establecidas (gráfica 1). 
Tabla 1. Clasificación de las muertes maternas según su causa. ${ }^{3}$

- Directa: aquélla ocurrida por complicaciones relacionadas con el embarazo y puerperio, por causa de una intervención, omisión y tratamiento incorrecto.

- Indirecta: la que resulta por el agravamiento de enfermedades preexistentes durante los cambios fisiológicos del embarazo.

- Fortuita: muerte por causa no relacionada con el embarazo o el puerperio.

En los países de Sudamérica el comportamiento epidemiológico en cuanto a mortalidad materna (expresada en muertes maternas por 100.000 nacidos vivos), si bien no es tan alto como en África, también es disímil. En países como Uruguay la cifra es relativamente baja (de 19), mientras que en otros, como Bolivia, alcanza a 390. En Colombia ocurren alrededor de 90 muertes maternas por 100.000 nacidos vivos. ${ }^{4}$

Esta elevada mortalidad en los países subdesarrollados de América Latina y África puede explicarse, entre otras causas, por las barreras a la educación en las mujeres, la pobreza, la inestabilidad socio-política y la elevada fertilidad en las mujeres de estas regiones. ${ }^{1}$ Sin embargo, algunos países como Kazajistán y Sri Lanka, clasificados como subdesarrollados, han establecido políticas y acciones de salud para detener las complicaciones maternas, y por eso hoy en día cuentan con bajos índices de mortalidad. ${ }^{5}$

Se ha determinado además que la falta de control prenatal en pacientes que han tenido abortos y partos realizados por personas mal entrenadas son factores predisponentes para complicaciones maternas. ${ }^{5,6}$

El término "casi pérdida", se refiere entonces a una disfunción orgánica aguda, desencadenada durante el embarazo y que pone en peligro la vida de la madre o el feto, que, si no se trata rápida y adecuadamente, puede finalizar en la muerte de la paciente. ${ }^{7}$

En un análisis comparativo y reciente de las casi pérdidas o near-misses realizado en Londres, se postula que la proporción muerte/near-miss es de 1:118, es decir 1 muerte por cada 118 casos de casi pérdida. ${ }^{7}$

\section{FACTORES PREDICTORES}

Existen predictores de morbilidad materna severa que son importantes de destacar, debido a que se ha establecido que por cada caso de muerte puede haber hasta 30 casos de morbilidad. Tales factores son la edad mayor de 34 años, raza no blanca, exclusión social, historia previa de hemorragia posparto, embarazo múltiple, hipertensión, diabetes mellitus, cesárea de emergencia y anemia. ${ }^{8}$

Algunas de las causas más comunes de mortalidad son prevenibles y aunque son similares en las diferentes regiones del mundo, el orden causal difiere de país en país. En el mundo las principales causas de mortalidad materna, en orden son: hemorragia, causas indirectas, sepsis, abortos inseguros, eclampsia, otras causas directas, parto obstruido e hipertensión. ${ }^{1}$

En Estados Unidos el embolismo encabeza la lista, seguido por las hemorragias, preeclampsia y eclampsia, infecciones, cardiomiopatía y anestesia. $^{1}$

En Colombia las principales causas de mortalidad son consecuencia de los trastornos hipertensivos

Gráfica 1. Estadísticas de mortalidad en algunos países; se observa una relación con situaciones socioeconómicas claramente establecidas. ${ }^{4}$

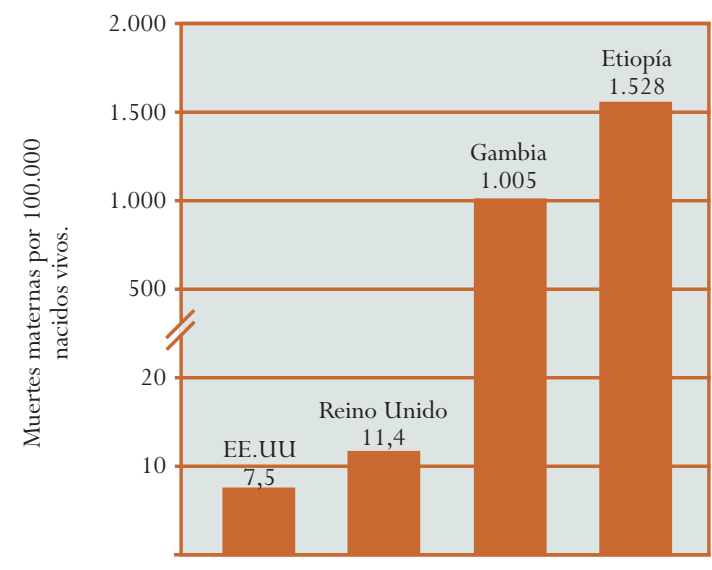


$(28,8 \%)$, complicaciones relacionadas con el parto $(17,2 \%)$, complicaciones del puerperio (8\%), abortos $(8,7 \%)$, y otras causas no especificadas (36\%). ${ }^{9}$

En los países desarrollados donde la mortalidad materna tiende a disminuir, es difícil formular guías de manejo basadas exclusivamente en los resultados de investigaciones con muestras pequeñas; por tal razón, hacer un seguimiento a las maternas con enfermedades que ponen en riesgo su vida (near-misses), podría ser útil para diseñar protocolos de manejo y prevenir la mortalidad en países subdesarrollados, o disminuir cada vez más las tasas de mortalidad en los desarrollados. Además permitiría la evaluación de otras necesidades, como la de sitios que permitan el cuidado perioperatorio óptimo para las gestantes, ya sea en unidades de cuidados intensivos o en unidades de alta dependencia obstétrica.

\section{ATENCIÓN EN UNIDAD DE CUIIDADOS INTENSIVOS}

Por la complejidad de los cuadros clínicos y la comorbilidad de las pacientes gestantes y posparto, es necesario que los tratamientos se continúen en las unidades de cuidados intensivos (UCI). La admisión a UCI de gestantes fluctúa entre $0,1 \%$ a $0,9 \%$. Hay varias causas de admisión a la UCI, siendo la más relevante la insuficiencia respiratoria. La mortalidad reportada de la paciente obstétrica críticamente enferma alcanza incluso cifras del 36\%. ${ }^{10}$

En un estudio realizado durante un período de 10 años en un hospital universitario de tercer nivel en Sao Paulo, Brasil, ${ }^{10}$ se encontró que la mortalidad de las maternas que requirieron tratamiento en UCI fue del 33,8\%. El análisis incluyó información proveniente de 28.660 gestantes posparto de las cuales $40(0,14 \%)$, requirieron tratamiento en unidad de cuidados intensivos. La edad promedio fue de 27,4 años, y la mayoría eran multíparas. Las principales indicaciones para su transferencia a la UCI fueron en orden descendente: afecciones hipertensivas (41\%), hemorragia (15\%) y sepsis
(13\%). De las pacientes que ingresaron a UCI el $69 \%$ lo hicieron por causas obstétricas y el $79 \%$ por patologías posparto. La estancia promedio fue de 8,9 días con rango entre 1 y 120 días, la ventilación mecánica fue requerida en el 63\% de casos, con una duración promedio de 6,5 días.

Esta elevada cifra de mortalidad, refleja la calidad del cuidado materno, la población de alto riesgo y las dificultades en el acceso a los servicios de salud en un país como Brasil. Se propone además en este estudio, que tener en cuenta la evaluación de las pacientes en riesgo y la identificación de las enfermedades que ocasionarían la muerte (near-miss) al binomio madre-hijo, podría disminuir la mortalidad materna, generando la necesidad de establecer servicios obstétricos en los países subdesarrollados, servicios que hagan un seguimiento estricto de las maternas con alto riesgo de morir. ${ }^{10}$

\section{ENFERMEDAD MATERNA Y RIESGO PERINATAL}

Siempre que hablemos de morbimortalidad materna debemos desarrollar el tema de mortalidad perinatal. Un manejo adecuado de la gestante de alto riesgo se debe traducir en menor morbimortalidad perinatal. La mortalidad perinatal es un término que incluye las muertes fetales desde la semana 22 de gestación en Colombia y desde la semana 28 para la OMS. ${ }^{11}$

Toda enfermedad o evento que ponga en riesgo la vida de la madre es potencialmente mortal para el feto o el neonato. Hay factores de riesgo materno que aumentan la mortalidad perinatal, entre los que se citan: edad materna avanzada ( $>$ 35 años), fumar, nulípara, multíparas ( $>4$ ) y bajo nivel socioeconómico. ${ }^{12}$

En Malawi, en un estudio prospectivo de cesáreas, se encontró una mortalidad perinatal a las 72 horas del $11,2 \%$. Dicho evento se asoció significativamente con la ruptura uterina y el uso de una técnica anestésica general en lugar de anestesia espinal. ${ }^{13}$

En Estados Unidos se llevó a cabo un estudio analizando el abrupcio placentario y la mortalidad perinatal. Dicho evento se presentó en 6,5 de cada 
1.000 embarazos y la mortalidad perinatal fue de 119 de cada 1.000 nacidos vivos, comparada con una mortalidad de 8,2 de cada 1.000 nacidos vivos sin abrupcio placentario. El abrupcio placentario está asociado a parto pretérmino, que a su vez se relaciona con bajo peso al nacer, los cuales aumentan el riesgo de mortalidad perinatal. Por tal razón se controlaron dichas variables, y sin embargo la asociación entre el abrupcio placentario y la mortalidad perinatal continuó siendo estadísticamente significativa, encontrándose que este evento obstétrico aumenta el riesgo de mortalidad perinatal en 25 veces. ${ }^{14}$

Estos estudios muestran claramente que un evento obstétrico hemorrágico aumenta el riesgo de una muerte perinatal así como una muerte materna.

\section{UNIDADES DE CUIDADOS INTENSIVOS, CUIDADOS ESPECIALES Y DE ALTA DEPENDENCIA}

El término de unidad de alta dependencia (UAD) es sinónimo de unidad de cuidados especiales (UCE). Las unidades obstétricas de Europa, en especial las del Reino Unido y España se han convertido en pioneros en cuanto al montaje y puesta en marcha de dichas unidades. La Alta Dependencia Obstétrica busca impactar en la morbimortalidad materna mediante el manejo adecuado y precoz de las gestantes críticamente enfermas y casi pérdidas ("near misses") sin tener que esperar el ingreso o la disponibilidad de cama en cuidados intensivos.

Las UCE o UAD, son lugares para pacientes que requieren monitoría no invasiva, soporte ventilatorio básico o no invasivo, y/o manejo de la falla de un sólo órgano. Proporcionan un cuidado intermedio entre la sala general y la UCI; a diferencia de la UCI, las pacientes allí ingresadas no requieren una relación tan alta entre enfermeras y médicos.

Cuidados intensivos (UCI) es el lugar donde llegan pacientes de todas las especialidades, incluyendo las gestantes que requieren observación permanente, tratamiento agresivo y continuo, y/o ventilación mecánica invasiva, además de otras indicaciones de soporte ventilatorio como fracción inspirada de oxígeno $\left(\mathrm{FIO}_{2}\right)$ mayor del 50\%, necesidad de terapia respiratoria más de dos veces al día y ventilación no invasiva y que requieren además soporte de dos o más órganos. Ingresan también a las UCI pacientes con enfermedades crónicas de uno o más órganos con descompensación aguda de uno y reversible de otro, y que necesitan el soporte de éste. Por la complejidad de las pacientes y de los tratamientos que se derivan, se debe disponer de una proporción alta de enfermeras profesionales y médicos especialistas durante las 24 horas del día. ${ }^{16,17}$

La duración de la estadía en la UCI y/o UAD de las embarazadas, comprende desde períodos cortos para resucitación, hasta varias semanas de cuidado intensivo y soporte orgánico. Además, con independencia del diagnóstico que origine el ingreso a estas unidades, lo que determina el pronóstico de la paciente es la calidad del cuidado (monitoría y personal adecuado). Las pacientes con riesgos conocidos de complicaciones no deben tener el parto en hospitales de maternidad sin UCI o UAD, a menos de que sean situaciones de emergencia, pero siempre procurando un adecuado sistema de remisión y transporte materno infantil. ${ }^{7}$

\section{TASAS DE INGRESO}

Diferentes estudios realizados en países desarrollados como Estados Unidos, Reino Unido, Francia, entre otros comprueban que los ingresos de pacientes embarazadas a la UCI son muy bajos. , $^{19,21}$

Panchal y cols. en el 2000 en un estudio que incluyó 822.591 pacientes que ingresaron para parto, determinaron que sólo $1.023(0,12 \%)$ ingresaron a UCI y de éstas únicamente fallecieron 34 (3,3\%). ${ }^{19}$

Loverro y cols. en Bari (Italia) encontraron que de 23.694 partos, sólo ingresaron a UCI 41 pacientes $(0,17 \%){ }^{20}$

No sólo es bajo el número de maternas que ingresan a UCI, sino también la relación de estas pacientes respecto a los ingresos totales a las unidades de cuidados intensivos, como lo demuestra 
un estudio realizado en el sur de Inglaterra: de 11.385 ingresos a UCI sólo 210 (1,84\%) fueron embarazadas, y estas 210 equivalen al 0,17\% de todos los partos. ${ }^{16}$

En un estudio realizado en India entre 1993 y 1998, 50 pacientes embarazadas ingresaron a la UCI, de un total de 26.986 partos, lo que equivale a 1 por cada 540 partos $(0,18 \%) .^{22}$

En los países desarrollados ha sido menor el número de embarazadas que requieren ingresar a unidades de cuidado intensivo con respecto a otros reportes, debido a la existencia de programas de vigilancia sobre esta población, y a que cada vez son más los centros que se ingresan en los estudios. Por el contrario, en los países en vías de desarrollo es mayor el ingreso a las unidades de cuidado intensivo, principalmente por la falta de cobertura en los programas de promoción y prevención sobre la población gestante.

Debido a que el ingreso de gestantes a la UCI es bajo, en estos servicios no suele contarse con la disponibilidad inmediata para su manejo; adicionalmente, la puntuación obtenida por estas pacientes en los índices de severidad (tales como el APACHE II) por lo regular es baja, y dado que éstos suelen usarse como un criterio aislado para definir el ingreso de las pacientes a la UCI, la embarazada será frecuentemente desplazada por otras pacientes con enfermedades crónicas asociadas, casos de emergencias relacionadas con trauma o postoperatorios de cirugías electivas. También, el no estar familiarizado con la atención de pacientes obstétricas, puede llevar al médico encargado de la UCI a preferir el ingreso de pacientes con enfermedades más afines con su formación básica de cuidado intensivo (por ejemplo, medicina interna, cirugía), motivo por el cual se evidencia la necesidad de un equipo multidisciplinario de obstetras, anestesiólogos, intensivistas y enfermeras profesionales para el adecuado manejo de las gestantes.

La carencia de cuidados intensivos en pacientes con indicaciones claras de éstos, aumenta considerablemente la mortalidad materna, ya que un ingreso temprano es fundamental para minimizar el deterioro y la falla orgánica determinantes importantes de la estancia posterior en UCI. Las pacientes necesitan manejo en UCI por complicaciones del embarazo, patologías no relacionas con el embarazo o enfermedades subyacentes que se empeoran durante el embarazo. Igualmente se requiere la evaluación previa de la paciente con riesgos por el grupo de trabajo multidisciplinario, incluido el intensivista, para no retardar su ingreso a la UCI. ${ }^{18}$

\section{CAUISAS DE INGRESO}

Las causas principales de ingreso a las unidades de cuidados intensivos son: preeclampsia, eclampsia, síndrome de HELLP, hemorragia obstétrica, hipertensión, postoperatorio de cesárea, enfermedad cardíaca, enfermedades pulmonares y procesos infecciosos. En algunos estudios se determinan otros factores tales como edad, edad gestacional, cirugías diferentes a cesárea, transfusiones, raza, estado civil y escolaridad, pero tienen una repercusión menor. Las principales causas de ingreso debido a preeclampsia son la necesidad de monitoreo hemodinámico invasivo (hipertensión severa y balance hídrico), ventilación mecánica (síndrome de dificultad respiratoria aguda, edema pulmonar, aspiración), protección de la vía aérea (convulsiones, edema de vía aérea superior), coagulación intravascular diseminada, síndrome de HELLP, falla renal aguda, neurológicas (convulsiones y accidente cerebro vascular). ${ }^{23}$

El trauma obstétrico es otra causa importante de ingreso a UCI y es una de las principales causas de mortalidad materna no obstétrica. Entre las lesiones que puede sufrir la gestante están: ruptura prematura de membranas ovulares, ruptura uterina y trauma fetal. A medida que avanza la gestación, se pueden presentar otros traumas asociados, tales como lesión visceral, abrupcio de placenta, lesión vesical y un mayor riesgo de ruptura uterina. ${ }^{23}$

En el estudio de Ryan las principales causas de ingreso a la UAD fueron: disfunción respiratoria, inestabilidad hemodinámica, compromiso 
neurológico y pacientes que sólo necesitaban observación. Ingresaron en total 123 pacientes que representan el 1,02\% de todos los partos atendidos en el hospital donde se realizó el estudio. ${ }^{24}$

En un estudio realizado en Francia por BouvierColle y cols en 1997, se encontraron como factores que aumentan la incidencia de ingreso a UCI, la falta de consulta prenatal, enfermedad subyacente grave y embarazo múltiple. ${ }^{25}$

En el reporte de Bari (Italia) la principal causa de ingreso a UCI es la preeclampsia con el 75,6\%, seguida por la- hemorragia obstétrica con el 14,7\% y como tercera y cuarta causa se presentan las enfermedades cardíacas con el $4,9 \%$ y pulmonares con $2,4 \% .^{20}$

Otro estudio realizado en Quebec (Canadá), muestra que la principal causa de ingreso a UCI es la hemorragia obstétrica (26\%) y la hipertensión (21\%). ${ }^{21}$ En un estudio realizado en el sur de Inglaterra la principal causa es la enfermedad hipertensiva del embarazo con $39,5 \%$, seguido de la hemorragia obstétrica con $33,3 \% .{ }^{16}$

En un reporte de la India la principal causa de ingreso a UCI fue shock en el $46 \%$ de las pacientes (28\% hemorrágico y 16\% séptico), seguida por complicaciones anestésicas (26\%) y la enfermedad hipertensiva del embarazo (18\%). La baja cifra de la enfermedad hipertensiva no necesariamente significa que esta patología sea infrecuente en la región, mientras que las complicaciones anestésicas son más altas que las de estudios en países desarrollados. ${ }^{22}$

Dentro de las causas no relacionadas con el embarazo, las enfermedades pulmonares son las más comunes y dentro de éstas el asma es la principal causa de ingreso a cuidados intensivos. Otros cuadros pulmonares son la embolia pulmonar, injuria pulmonar aguda y el edema agudo de pulmón. La injuria pulmonar aguda puede ser desencadenada por preeclampsia, hemorragia obstétrica, amnionitis, embolismo de líquido amniótico o endometritis. El edema pulmonar puede ser causado por preeclampsia, tocolisis y disfunción cardíaca.

\section{INDICACIONES DE VENTILACIÓN MECÁNICA Y MONITOREO INVASIVO}

Las indicaciones más comunes para el uso de ventilación mecánica son protección de la vía aérea, manejo de secreciones, manejo de hipoxia y falla respiratoria. Otra indicación de ventilación mecánica es la convulsión recurrente a pesar del manejo adecuado, las cuales son más comunes en las pacientes con eclampsia y en las primeras 24 horas posparto. ${ }^{23}$

El estudio de la India reportó que la ventilación mecánica fue necesaria en el 64\% de las pacientes que ingresaron a UCI y la ventilación no invasiva en el 24\% de los casos. ${ }^{22}$

El monitoreo hemodinámico invasivo está indicado principalmente para el manejo de la hipertensión severa y el balance de líquidos. Es el método ideal para este propósito y está indicado en pacientes con oliguria, edema pulmonar, hipertensión resistente, shock hipovolémico, disfunción o enfermedad cardíaca descompensada, cardiomiopatía con fracción de eyección menor del 40\%, disfunción pulmonar y disfunción renal. Reportes de países subdesarrollados, como India, muestran que $10 \%$ de las pacientes que ingresaron a UCI requieren monitoreo cardíaco y $60 \%$ transfusiones. $^{22}$

La medición invasiva de la presión arterial tiene las mismas indicaciones del catéter de arteria pulmonar. ${ }^{26,27} \mathrm{El}$ catéter de arteria pulmonar mide la presión venosa central, presiones sistólica y diastólica de dicha arteria, y la presión capilar pulmonar; además del cálculo de otras variables, como el gasto cardíaco, resistencias vasculares sistémica y pulmonar y otros parámetros hemodinámicos.

\section{DUIRACIÓN DE LA ESTANCIA}

Son pocos los estudios que hacen referencia a la estancia de las gestantes en la UCI. Entre ellos, la publicación de una investigación realizada en el sur de Inglaterra reporta que el promedio de días de estancia en UCI de las gestantes (menos de dos días) es inferior que el de la población no gestante. ${ }^{16} \mathrm{Se}$ 
encontró además que el 35\% de las pacientes que ingresaron a UCI no requirieron procedimientos específicos como ventilación mecánica, soporte inotrópico, monitoreo invasivo o diálisis, lo que se relaciona estrechamente con el tiempo de estancia. ${ }^{16}$ En otro reporte originado en Nueva Delhi, la estancia fue de 33,6 horas. ${ }^{22}$

G. Zakalik y cols. en Argentina reportaron los ingresos de pacientes obstétricas a UCI durante cuatro años. Sobre un total de 1.579 casos, se registraron 183 pacientes obstétricas, lo que correspondió al 0,42\% de la población obstétrica total ingresada al hospital (42.774 pacientes), y al $11,58 \%$ de los ingresos a UCI, durante el mismo período. El principal motivo de ingreso fue sepsis/ shock séptico (31,69\% de los casos), seguido de preeclampsia/eclampsia $(21,31 \%)$ e hipovolemia (9,84\%). El 65,5\% de la sepsis/shock séptico corresponde a aborto séptico.

Los diferentes sistemas de evaluación de severidad de la enfermedad como el APACHE II (Acute Physiological and Chronic Health Evaluation) y el SAPS II (Simplified Acute Physiology Score) son utilizados en la población gestante, pero debido a los cambios fisiológicos que ocurren normalmente durante el embarazo y a la ausencia de enfermedades crónicas que aumenten su puntaje, suministran resultados equívocos cuando se comparan con el resto de la población. Además, no permiten anticipar el riesgo de complicaciones, ni predicen estancias prolongadas. Su uso como indicadores de gravedad para definir la admisión de las pacientes a la UCI, hace que muchas no ingresen debido a que en la población obstétrica estos puntajes son mas bajos. Debido a lo anterior, en los diferentes reportes se sugiere desarrollar un sistema especial que determine adecuadamente la severidad en la materna. ${ }^{16,18,19}$

\section{MORTALIDAD}

\section{DE LA GESTANTE EN UCI}

En un reporte sobre muerte materna en el Reino Unido, entre 1991 y 1999, las causas directas fueron: tromboembolismo, desórdenes hiperten- sivos, embarazo temprano, embolismo de líquido amniótico, hemorragia, sepsis, misceláneas y trauma genitourinario. Las causas indirectas fueron cardíacas, neurológicas, infecciosas, psiquiátricas, respiratorias, endocrinas, metabólicas, circulatorias y hematológicas. ${ }^{16}$

En el Reino Unido la mortalidad materna estimada fue del 3,3\%. Esta baja mortalidad refleja un adecuado trabajo interdisciplinario en la UCI. ${ }^{15}$

En el reporte del trienio del Reino Unido (19971999), se presentaron 119 muertes maternas en UCI. Estas se clasificaron en directas (53\%): tromboembolismo, enfermedad hipertensiva del embarazo, hemorragia, embolismo de líquido amniótico, embarazo temprano, sepsis, anestésicas y otras. Las causas indirectas (35\%): cardíacas, psiquiátricas. $^{7}$

La mortalidad materna en la India, en pacientes admitidas a UCI fue del $28 \%$, siendo las principales causas coagulación intravascular diseminada y el shock séptico, seguido por shock hemorrágico, embolismo pulmonar y falla hepatorrenal secundaria a eclampsia. Esta tasa de mortalidad es 8 veces más alta que la demostrada en países desarrollados. ${ }^{22}$

\section{UINIDADES DE ALTA DEPENDENCIA}

En el Reino Unido debido a las bajas tasas de ingreso de las embarazadas a UCI, su corta estancia, poco requerimiento de procedimientos de UCI y la baja mortalidad, se han desarrollado las unidades de alta dependencia (UAD) para el manejo de la gestante crítica. En caso de ser necesario un procedimiento de UCI la paciente se traslada a ésta. Las UAD son atendidas por un anestesiólogo y un obstetra permanentemente y están cerca al área quirúrgica.

Además, la implementación de unidades de alta dependencia, demostró que la utilización de la UCI bajó de $0,08 \%$ a 0,04\%. Este estudio concuerda con estudios previos que concluyen que la intervención y tratamiento temprano de la paciente embarazada de alto riesgo disminuye la necesidad de ingresar a la UCI y la gran mayoría se puede tratar en la UAD. ${ }^{24}$ 
El estudio del sur de Inglaterra también concluye, basado en los datos antes anotados, que las pacientes embarazadas en estado crítico pueden ser vigiladas y manejadas de manera temprana y de esta manera prevenir y controlar complicaciones, hospitalizaciones y estancias prolongadas en UCI. ${ }^{16}$

En el último reporte de mortalidad materna del Reino Unido, "Why mothers die? 2000 - 2002. (Confidential Enquiry into Maternal and Child Health) CEMACH", al analizar el tema de admisiones a UCI, los autores resaltan varios puntos: menos de 1 de cada 1.000 madres ingresaron a éstas; el mayor número estuvo representado por población joven sin enfermedades asociadas, quienes, en caso de morir, generan un importante trauma no sólo a las familias, sino también a la sociedad y al personal asistencial de cuidado intensivo. Señalan cómo la población obstétrica críticamente enferma se ha convertido en donante potencial de órganos debido al aumento de complicaciones hipertensivas, hemorragia cerebral y muerte cerebral.

Otro punto importante que realza este reporte es que el hecho de no tener disponibilidad de cama en cuidados intensivos no exime al grupo de tratamiento (anestesiólogos, obstetras) de realizar las medidas iniciales de resucitación, monitoría invasiva, soporte ventilatorio mecánico y corrección de anomalías en la coagulación, ácido-base, electrolitos o metabólicas. ${ }^{28}$

Las UAD pueden estar ubicadas en hospitales regionales que no tienen UCI, pero que sí cuentan con anestesiólogos y obstetras entrenados en cuidado crítico o con los conceptos claros de reanimación inicial de las gestantes de alto riesgo, estableciendo cuáles pacientes se pueden manejar en estas áreas, y en ocasiones evitando traslados innecesarios a las unidades de cuidados intensivos, o permitir su manejo adecuado inicialmente, mientras se define el traslado a UCI.

Debido al bajo número de ingresos de embarazadas a UCI, el equipo médico y paramédico puede no estar familiarizado con ellas, no tener conocimiento amplio de los cambios fisiológicos que se producen en el embarazo, los efectos hemodinámicos posparto, efectos de drogas en la circulación placentaria, y contar con poca evidencia de las intervenciones y tratamientos más adecuados. ${ }^{17}$

Para un correcto manejo de la paciente embarazada crítica es necesario un equipo multidisciplinario que tenga conocimiento y experiencia en el manejo de estas pacientes, criterios claramente definidos para el ingreso a UCI o UAD, protocolos de manejo específicos para las entidades propias de las gestantes y una adecuada red interinstitucion al que permita el transporte ágil entre las unidades de los centros hospitalarios cercanos disponibles.

\section{PANORAMA EN COLOMBIA}

En nuestro contexto surge el siguiente interrogante: ¿están entrenados los equipos obstétricos de Colombia para el manejo de una paciente embarazada críticamente enferma?

En el mundo existen cursos que específicamente tratan de cubrir estos aspectos; son dictados por obstetras y anestesiólogos que laboran en servicios obstétricos (tabla 2).

Hasta ahora en nuestro medio se han realizado pocos esfuerzos a gran escala para la capacitación de equipos obstétricos en gestantes críticamente enfermas, a pesar de que el gobierno ha implementado medidas para impactar a corto plazo la mortalidad materna, como el "Plan de Choque“, del Ministerio de

\section{Tabla 2. Algunos de los cursos de entrenamiento más reconocidos para equipos obstétricos}

- Advanced Cardiac Life Support. ACLS for Experienced Providers. Cardiac Arrest Associated with Pregnancy. American Heart Association. 2003. USA.

- Managing Obstetric Emergencies and Trauma. MOET. Royal College of Obstetricians and Gynecologists 2003. UK.

- Multidisciplinary Obstetric Simulated Emergency Scenarios. MOSES NHS.

- Advanced Life Support in Obstetrics. ALSO College of Family Physicians. USA.

- Averting Maternal Death and Disability. AMDD Columbia University. USA. 
la Protección social, Republica de Colombia (posible de consultar en www.minproteccionsocial.gov.co).

Actualmente se trabaja en la creación y apertura de unidades de alta dependencia obstétrica en el país, ejemplos de esto son los proyectos en la Clínica del Prado y la Clínica Universitaria Bolivariana en la ciudad de Medellín.

El objetivo básico de las unidades de alta dependencia obstétrica debe ser el de proporcionar apoyo a las gestantes críticamente enfermas que permita optimizar el tiempo de quirófanos y salas de trabajo de parto para que estos no colapsen ante la llegada de una paciente compleja, y servir como puente a las unidades de cuidado intensivo, cuando la severidad de la gestante permita anticipar una estancia mayor a 48 horas en alta dependencia, permitiendo la implementación de medidas de monitoria invasiva y reanimación inicial para no perder tiempo en esas horas iniciales, que manejadas adecuadamente impactarán favorablemente el pronóstico de las gestantes.

\section{ABORDAJE DEL}

\section{PROBLEMA Y SUIGERENCIAS}

- La proporción de camas ocupadas en UCI por pacientes obstétricas es baja, los motivos de esta situación y si debe existir una UCI exclusivamente para esta población deben ser parte de agendas de trabajo e investigaciones orientadas a este tópico.

- Cuando las gestantes esperan la disponibilidad de cama en cuidado intensivo se pierden horas valiosas del manejo inicial, que contribuyen a la disfunción de múltiples órganos, a la estancia prolongada posterior en UCI y, pueden contribuir a la muerte de la gestante y el feto.

- En países desarrollados, la implementación de unidades de alta dependencia obstétrica (UAD) ha sido benéfica y ha impactado favorablemente en la disminución de la morbimortalidad materna y perinatal.

- El desarrollo de unidades de alta dependencia obstétrica en nuestro medio, permitirían manejar las primeras horas de estas pacientes críticamente enfermas sin colapsar las unidades de atención materna en su funcionamiento y definir con criterios específicos si las gestantes deben permanecer en estas o deben ser trasladadas a cuidados intensivos

- Nuestros equipos multidisciplinarios de manejo obstétrico se deben entrenar para el manejo de pacientes obstétricas críticamente enfermas, en especial manejo de emergencias y tratamiento inicial en las fases tempranas de descompensación.

\section{REFERENCIAS}

1. Tracy EE, Tomich PG. Maternal mortality: an international crisis. ACOG Clin Rev 2002;7:13-6.

2. Hawkins JL. Maternal mortality: anesthetic implications. Int Anesthesiol Clin 2002;40:1-11.

3. Wagaarachchi PT, Fernando L. Trends in maternal mortality and assessment of substandard care in a tertiary care hospital. Eur J Obstet Gynecol Reprod Biol 2002;101:36-40.

4. Cuartas J, Mesa C. Factores asociados a mortalidad maternal. Medellín 2001-2003. Medellín: Ed Marín Vieco; 2004.

5. Khosla AH, Dahiya K, Sangwan K, Maternal mortality and "near-miss" in rural north India. Int J Gynecol Obstet 2000;68:163-4.

6. Mantel GD, Buchmann E, Rees H, Pattinson RC. Severe acute maternal morbidity: a pilot study of a definition for a near-miss. Br J Obstet Gynecol 1998;105:985-90.

7. Crowhurst JA, Plaat F. Why mothers die-report on confidential enquiries into maternal deaths in the United Kingdom 1994-96. Anaesthesia 1999;54:207-9.

8. Paruk F, Moodley J. Severe obstetric morbidity. Curr Opin Obstet Gynecol 2001;13:563-8.

9. Instituto Nacional de Salud, Vol 6, No 23, dic 2001.

10. Dias de Souza JP, Duarte G, Basile-Filho A. Near-miss maternal mortality in developing countries. Eur J Obstet Gynecol Reprod Biol 2002;104:80.

11. Kramer MS, Liu S, Luo Z, Yuan H, Platt RW, Joseph KS, et. al. Analysis of perinatal mortality and its components: time for a change? Am J Epidemiol 2002;156:493-7.

12. Winbo I, Serenius F, Dahlquist G, Kallen B. Maternal risk factors for cause-specific stillbirth and neonatal death. Acta Obstet Gynecol Scand 2001;80:235-44. 
13. Fenton PM, Whitty CJ, Reynolds F. Caesarean section in Malawi: prospective study of early maternal and perinatal mortality. BMJ 2003;327:587.

14. Ananth CV, Wilcox AJ. Placental abruption and perinatal mortality in the United States. Am J Epidemiol 2001;153:332-7.

15. Pacora P, Huiza L, Maymon E. Maternal stature lower than $160 \mathrm{~cm}$ identifies $90 \%$ of cases of maternal and perinatal mortality/morbidity in Lima, Peru. Am J Obstet Gynecol 2002;187 (6 part 2) Suppl 143.

16. Hazelgrove JF, Price C, Pappachan VJ, Smith GB. Multicenter study of obstetric admissions to 14 intensive care units in Southern England. Crit Care Med 2001;29:770-5.

17. Yentis SM. Maternal morbidity and mortality - obstetric intensive care. Obstetric Gynecology. Royal College of Obstetricians and Gynecologists. London: RCOG press; 2002.

18. James MFM, Anthony J. Critical care management of the pregnant patient. En: Birnbach DJ, Gatt SP, Datta S. Textbook of obstetric anesthesia. Philadelphia, Penn: Edit Churchill Livingstone; 2000. p. 716-32.

19. Panchal S, Arria AM, Harris AP. Intensive care utilization during hospital admission for delivery: prevalence, risk factors, and outcomes in a statewide population. Anesthesiology 2000 ;92:1537-44.

20. Loverro G, Pansini V, Greco P, Vimercati A, Parisi AM, Selvaggi L. Indications and outcome for intensive care unit admission during puerperium. Arch Gynecol Obstet 2001;265:195-8.

21. Mahutte NG, Murphy-Kaulbeck L, Le Q, Solomon J, Benjamin A, Boyd ME. Obstetric admissions to the intensive care unit. Obstet Gynecol 1999;94:263-6.

22. Tripathi R, Rathore AM, Saran S. Intensive care for critically ill obstetric patients. Int J Gynaecol Obstet 2000;68:257-8.

23. Dhond GR, Dob DP. Intensive care of the obstetric patient. Curr Anaesth Crit Care 2000;11:1-6.

24. Ryan M, Hamilton V, Bowen M, McKenna P. The role of a high-dependency unit in a regional obstetric hospital. Anaesthesia 2000;55:1155-8.

25. Bouvier-Colle MH, Varnoux N, Salanave B, Ancel PY, Breart G. Case-control study of risk factors for obstetric patients, admission to intensive care units. Europ J Obstetric Gynecol Reprod Biol 1997;74:173-7.

26. Burton R, Belfort M, Anthony J. Management of the Pregnant ICU Patient. Clin Pulmonary Med 2002;9:87-96.

27. Nolan TE, Wakefield ML, Devoe LD. Invasive hemodynamic monitoring in obstetrics. A critical review of its indications, benefits, complications, and alternatives. Chest 1992;101:1429-33.

28. Why mothers die? 2000-2002. Confidential enquiry into maternal and child health. CEMACH RCOG Press; 2004.

Conflicto de intereses: ninguno declarado. 